\title{
Edge Domination Number of Corona Product Graph of a Cycle with a Star
}

\author{
J. Sreedevi \\ J.L.in Mathematics, \\ A.P.R.J.C., \\ BANAVASI, Kurnool (Dt) \\ A. P., India
}

\author{
B. Maheswari \\ Dept. of Applied Mathematics, \\ Sri Padmavathi Mahila \\ Viswavidyalayam, \\ Tirupati, A.P., India
}

\begin{abstract}
Graph Theory has been realized as one of the most useful branches of Mathematics of recent origin with wide applications to combinatorial problems and to classical algebraic problems. Graph theory has applications in diverse areas such as social sciences, linguistics, physical sciences, communication engineering etc.
\end{abstract}

The theory of domination in graphs is an emerging area of research in graph theory today. It has been studied extensively and finds applications to various branches of Science \& Technology. An introduction and an extensive overview on domination in graphs and related topics is surveyed and detailed in the two books by Haynes et al $[7,8]$.

Products are often viewed as a convenient language with which one can describe structures, but they are increasingly being applied in more substantial ways. Every branch of mathematics employs some notion of product that enables the combination or decomposition of its elemental structures.

In this paper some results on minimal edge dominating sets of corona product graph of cycle with a star are discussed.

\section{Keywords}

Corona Product, edge dominating set, edge domination number.

\section{INTRODUCTION}

Domination Theory has a wide range of applications to many fields like Engineering, Communication Networks, Social sciences, linguistics, physical sciences and many others. Allan, R.B. and Laskar, R.[1], Cockayne, E.J. and Hedetniemi, S.T. [4] have studied various domination parameters of graphs.

Frucht and Harary [6] introduced a new product on two graphs $\mathrm{G} 1$ and $\mathrm{G} 2$, called corona product denoted by $\mathrm{G} 1 \odot \mathrm{G} 2$. The object is to construct a new and simple operation on two graphs G1 and G2 called their corona, with the property that the group of the new graph is in general isomorphic with the wreath product of the groups of G1 and of G2 .

The concept of edge domination was introduced by Mitchell and Hedetniemi [11] and it is explored by many researchers. Arumugam and Velammal [3] have discussed the edge domination in graphs while the fractional edge domination in graphs is discussed in Arumugam and Jerry [2]. The complementary edge domination in graphs is studied by Kulli and Soner [10] while Jayaram [9] has studied the line dominating sets and obtained bounds for the line domination number. The bipartite graphs with equal edge domination number and maximum matching cardinality are characterized by Dutton and Klostermeyer [5] while Yannakakis and Gavril
[12] have shown that edge dominating set problem is NPcomplete even when restricted to planar or bipartite graphs of maximum degree. The edge domination in graphs of cubes is studied by Zelinka [13].

\section{CORONA PRODUCT GRAPH $C_{n} \odot K_{1, m}$ AND ITS PROPERTIES}

The corona product of a cycle $C_{n}$ wirh a star graph $K_{1, m}$ for $\mathrm{m} \geq 2$, is a graph obtained by taking one copy of a $n$-vertex graph $C_{n}$ and n copies of $K_{1, m}$ and then joining the $\mathrm{i}^{\text {th }}$ vertex of $C_{n}$ to all vertices of $\mathrm{i}^{\text {th }}$ copy of $K_{1, m}$. This graph is denoted by $C_{n} \odot K_{1, m}$.

Now we discuss some properties of the corona product graph $G=C_{n} \odot K_{1, m}$.

Theorem 2.1: The graph $G=C_{n} \odot K_{1, m} \quad$ is a connected graph.

Proof: Consider the graph $G=C_{n} \odot K_{1, m}$.

By the definition of the corona product graph $\mathrm{G}$, the $\mathrm{i}^{\text {th }}$ vertex of $C_{n}$ is adjacent to each vertex of $\mathrm{i}^{\text {th }}$ copy of $K_{1, m}$ in $\mathrm{G}$. That is the vertices in $C_{n}$ are connected to the vertices of $K_{1, m}$. Since $C_{n}$ and $K_{1, m}$ are connected, it follows that $\mathrm{G}$ is connected.

Hence $G=C_{n} \odot K_{1, m}$ is a connected graph.

Theorem 2.2: The degree of a vertex $v_{i}$ in $G=C_{n} \odot K_{1, m}$ is given by

$d\left(v_{i}\right)= \begin{cases}m+3, & \text { if } v_{i} \in C_{n}, \\ m+1, & \text { if } v_{i} \in K_{1, m} \text { and } v_{i} \text { is in first partition, } \\ 2, & \text { if } v_{i} \in K_{1, m} \text { and } v_{i} \text { is in second partition. }\end{cases}$

Proof: Consider the graph $G=C_{n} \odot K_{1, m}$.

By the definition of the graph $\mathrm{G}$, the $i^{\text {th }}$ vertex of $C_{n}$ is joined to $\mathrm{m}+1$ vertices of $i^{\text {th }}$ copy of $K_{1, m}$ in G. The vertex $v_{i} \in C_{n}$ is adjacent to two vertices of $C_{n}$. Therefore the degree of a vertex $v_{i} \in C_{n}$ is $\mathrm{m}+3$.

Therefore $d\left(v_{i}\right)=m+3$, if $v_{i} \in C_{n}$.

There are $m+1$ vertices in each copy of $K_{1, m}$, such that one vertex $v$ of $K_{1, m}$ is of degree $m$ and $m$ vertices of $K_{1, m}$ are of degree one. Since these vertices are adjacent to a corresponding vertex of $C_{n}$ in $\mathrm{G}$, it follows that, the degree of a vertex $v \in K_{1, m}$ in $\mathrm{G}$ is

$d\left(v_{i}\right)=\left\{\begin{array}{c}m+1, \text { if } v_{i} \in K_{1, m} \text { and } v_{i} \text { is in first partition, } \\ 2, \text { if } v_{i} \in K_{1, m} \text { and } v_{i} \text { is in second partition. }\end{array}\right.$ 
Theorem 2.3: The number of vertices and edges in $G=$ $C_{n} \odot K_{1, m}$ is given respectively by

$$
\begin{aligned}
& \text { 1. }|V(G)|=n(m+2), \\
& \text { 2. }|E(G)|=2 n(m+1) .
\end{aligned}
$$

Proof: Consider the graph $G=C_{n} \odot K_{1, m}$ whose vertex set is denoted by $V$.

By the definition, the vertex set of $\mathrm{G}$ contains the vertices of $C_{n}$ and the vertices of $K_{1, m}$ in $n$ - copies. As there are $n$ vertices in $C_{n}$ and $m+1$ vertices in $K_{1, m}$, it follows that

$|V(G)|=n+n(m+1)=n(m+2)$.

By Theorem 2.2., the degree of a vertex $v_{i} \in G$ is given by $d\left(v_{i}\right)=\left\{\begin{array}{l}m+3, \text { if } v_{i} \in C_{n}, \\ m+1, \text { if } v_{i} \in K_{1, m} \text { and } v_{i} \text { is in first partition, } \\ 2, \quad \text { if } v_{i} \in K_{1, m} \text { and } v_{i} \text { is in second partition }\end{array}\right.$

$$
\text { Hence } \begin{aligned}
& |E(G)|=\frac{1}{2}\left(\sum_{v_{i} \in C_{n}} \operatorname{deg}\left(v_{i}\right)+n \sum_{v_{i} \in K_{1, m}} \operatorname{deg}\left(v_{i}\right)\right) \\
= & \frac{1}{2}(n(m+3)+n((m+1)+\underbrace{2+2+\ldots . .+2}_{m \text {-times }})) \\
= & \frac{1}{2}(n(m+3)+n(m+1)+2 n m) \\
= & \frac{1}{2}(n(m+3+m+1+2 m)) \\
= & \frac{1}{2}(n(4 m+4))=2 n m+2 n \\
= & 2 n(m+1) . \mathbf{a}
\end{aligned}
$$

Theorem 2.4: The graph $G=C_{n} \odot K_{1, m}$ is not eulerian.

Proof: In Theorem 2.2., we have seen that the degree of a vertex $v_{i}$ in $G$ has three different values viz., $m+3, m+1$ and 2, if $v_{i} \in C_{n}$ or $v_{i} \in K_{1, m}$. It is apparent that for any value of $m$, the degree of the vertex $v_{i}$ is not even.

Therefore $G$ is not eulerian.

Theorem 2.5: The graph $G=C_{n} \odot K_{1, m}$ is non - hamiltonian.

Proof: Let $V$ denote the vertex set of the graph $G=C_{n} \odot K_{1, m}$. Let us denote the vertices of $C_{n}$ by $v_{1}, v_{2}, \ldots \ldots, v_{n}$ and denote the vertices of $i^{t h}$ copy of $K_{1, m}$, by $u_{i 0}, u_{i 1}, u_{i 2}, \ldots \ldots \ldots, u_{i m}$.

In $G$, the $i^{\text {th }}$ vertex of $C_{n}$ is adjacent to each vertex of $i^{\text {th }}$ copy of $K_{1, m}$.

Then $v_{i}, u_{i 1}, u_{i 2}, \ldots \ldots \ldots, u_{i m}, v_{i}$ form a cycle in $G$.

In this way, we have $n$ disjoint cycles in $G$, and these cycles are formed at each vertex of $C_{n}$. Since the vertices of $C_{n}$ are in a cycle, we can not find a closed path in $G$ that contains all the vertices of $G$.

Therefore $G=C_{n} \odot K_{1, m}$ is non- hamiltonian.

Theorem 2.6: The graph $G=C_{n} \odot K_{1, m}$ is not bipartite.

Proof: Consider the vertex $v_{i} \in C_{n}$ and the vertices $u_{i 0}, u_{i 1}, u_{i 2}, \ldots \ldots \ldots, u_{i m}$ in the $i^{t h}$ copy of $K_{1, m}$ in G. Obviously the vertices $v_{i}, u_{i 0}$ and $u_{i 1}$ form an odd cycle.

Therefore the graph $G=C_{n} \odot K_{1, m}$ is not bipartite.

\section{EDGE DOMINATING SETS OF $C_{n} \odot K_{1, m}$}

In this section, edge dominating sets of the graph

$G=C_{n} \odot K_{1, m}$ are discussed. Also edge domination number of this graph is obtained. First we recall some definitions.

Definition: Let $G(V, E)$ be a graph. A subset $\mathrm{D}$ of $\mathrm{E}$ is said to be an edge dominating set(EDS) of $G$ if every edge in $E$ - D is adjacent to some edge in D. An edge dominating set $\mathrm{D}$ is called a minimal edge dominating set (MEDS) if no proper subset of $\mathrm{D}$ is an edge dominating set of $\mathrm{G}$.

Definition: The edge domination number of $G$ is the minimum cardinality taken over all minimal edge dominating sets in $\mathrm{G}$ and is denoted by $\gamma^{\prime}(G)$.

The vertices and the edges in $C_{n}$ are denoted by $v_{1}, v_{2}, \ldots \ldots \ldots, v_{n}$ and $e_{1}, e_{2}, \ldots \ldots, e_{n}$ respectively where $e_{i}$ is the edge joining the vertices $v_{i}$ and $v_{i+1}, \mathrm{i} \neq \mathrm{n}$. For $\mathrm{i}=\mathrm{n}, e_{n}$ is the edge joining the vertices $v_{n}$ and $v_{1}$.

The vertex in the first partition of $i^{\text {th }}$ copy of $K_{1, m}$ is denoted by $u_{i}$ and the vertices in the second partition of $i^{t h}$ copy of $K_{1, m}$ are denoted by $w_{i 1}, w_{i 2}, \ldots \ldots \ldots, w_{i m}$. The edges in the $i^{t h}$ copy of $K_{1, m}$ are denoted by $l_{i j}$ where $l_{i j}$ is the edge joining the vertex $u_{i}$ to the vertex $w_{i j}$. There are another type of edges, denoted by $h_{i}, h_{i j}$. Here $h_{i}$ is the edge joining the vertex $v_{i}$ in $C_{n}$ to the vertex $u_{i}$ in the $i^{\text {th }}$ copy of $K_{1, m}$. The edge $h_{i j}$ is the edge joining the vertex $v_{i}$ in $C_{n}$ to the vertex $w_{i j}$ in the $i^{t h}$ copy of $K_{1, m}$.

We denote the edge induced sub graph on the set of edges $E_{i}=\left\{h_{i}, h_{i j}, l_{i j}: j=1,2, \ldots \ldots ., m\right\}$ by $H_{i}$, for $\mathrm{i}=1,2, \ldots \ldots ., \mathrm{n}$.

We now prove some results in the corona product graph

$G=C_{n} \odot K_{1, m}$.

Theorem 3.1: The adjacency of an edge e in $G=C_{n} \odot$ $K_{1, m}$ is given by

$\operatorname{adj}(e)= \begin{cases}2 m+4, & \text { if } e=e_{i} \in C_{n}, \\ m+1, & \text { if } e \in i^{t h} \text { copy of } K_{1, m}, \\ 2 m+2, & \text { if } e=h_{i} \in H_{i}, \\ m+3, & \text { if } e=h_{i j} \in H_{i} .\end{cases}$

Proof: Consider the graph $G=C_{n} \odot K_{1, m}$.

By the definition of the graph $\mathrm{G}$, the $i^{\text {th }}$ vertex of $C_{n}$ is joined to $\mathrm{m}+1$ vertices of the $i^{\text {th }}$ copy of $K_{1, m}$ in $\mathrm{G}$.

Case 1: Let $e=e_{i} \in C_{n}$.

The edge $e=e_{i} \in C_{n}$ is adjacent to two edges of $C_{n}$ viz., $e_{i-1}, e_{i+1}$ when $\mathrm{i} \neq 1, \mathrm{n} ; e_{2}, e_{n}$ when $\mathrm{i}=1 ; e_{1}, e_{n-1}$ when $\mathrm{i}=\mathrm{n}$; $\mathrm{m}+1$ edges joining the vertex $v_{i}$ in $C_{n}$ to the vertices of $H_{i}$ viz., $h_{i}, h_{i 1}, h_{i 2}, \ldots \ldots \ldots . . . ., h_{i m} ; \mathrm{m}+1$ edges joining the vertex $v_{i+1}$ in $C_{n}$ to the vertices of $H_{i+1}$ viz., $h_{i+1}, h_{(i+1) 1}, h_{(i+1) 2}, \ldots \ldots \ldots ., h_{(i+1) m}$.

Therefore $\operatorname{adj}(\mathrm{e})=$ number of edges adjacent to $\mathrm{e}$

$$
=2+\mathrm{m}+1+\mathrm{m}+1=2 \mathrm{~m}+4 .
$$

Case 2: Let $e=l_{i j} \in H_{i}$ where $l_{i j}$ is the edge joining the vertex $u_{i}$ in $H_{i}$ to the vertex $w_{i j}$ in $H_{i}$. The edge $e=l_{i j}$ is adjacent to $\mathrm{m}-1$ edges joining the vertex $u_{i}$ in $H_{i}$ to the vertices $w_{i 1}, w_{i 2}, \ldots \ldots \ldots \ldots \ldots, w_{i(j-1)}, w_{i(j+1)}, \ldots \ldots \ldots, w_{i m}$ in $H_{i}$ viz., $\quad l_{i 1}, l_{i 2}, \ldots \ldots \ldots, l_{i(j-1)}, l_{i(j+1)}, \ldots \ldots . ., l_{i m} ; \quad$ the edge 
joining the vertex $v_{i}$ in $C_{n}$ to the vertex $u_{i}$ in $H_{i}$ viz., $h_{i}$; the edge joining the vertex $v_{i}$ in $C_{n}$ to the vertex $w_{i j}$ in $H_{i}$ viz., $h_{i j}$.

Then $\operatorname{adj}(\mathrm{e})=$ number of edges adjacent to $\mathrm{e}$

$$
=m-1+1+1=m+1 .
$$

Case 3: Let $\mathrm{e}=h_{i} \in H_{i}$ where $\mathrm{i}=1,2, \ldots \ldots \ldots, \mathrm{n}$ and $h_{i}$ is the edge joining the vertex $v_{i}$ in $C_{n}$ to the vertex $u_{i}$ in $H_{i}$. Now the edge $\mathrm{e}=h_{i}, \mathrm{i} \neq 1$ is adjacent to two edges in $C_{n}$ viz., $e_{i-1}$, $e_{i}$ and the edge $h_{1}$ is adjacent to two edges in $C_{n}$ viz., $e_{1}, e_{n}$; m edges joining the vertex $v_{i}$ in $C_{n}$ to the vertices in $H_{i}-\left\{v_{i}\right\}$ viz., $h_{i 1}, h_{i 2}, \ldots \ldots \ldots, h_{i m} ; \mathrm{m}$ edges joining the vertex $u_{i}$ in $H_{i}$ to the vertices $w_{i 1}, w_{i 2}, \ldots \ldots \ldots, w_{i m}$ in $H_{i}$ viz., $l_{i 1}, l_{i 2}, \ldots . ., l_{i m}$.

Then $\operatorname{adj}(e)=$ number of edges adjacent to e

$$
=2+\mathrm{m}+\mathrm{m}=2 \mathrm{~m}+2 .
$$

Case 4: Let $\mathrm{e}=h_{i j} \in H_{i}$ where $\mathrm{i}=1,2, \ldots \ldots \ldots, \mathrm{n} ; \mathrm{j}=1,2, \ldots \ldots, \mathrm{m}$ and $h_{i j}$ is the edge joining the vertex $v_{i}$ in $C_{n}$ to the vertex $w_{i j}$ in $H_{i}$. Now the edge e $=h_{i j}$ is adjacent to two edges in $C_{n}$ viz., $e_{i-1}, e_{i}$ when $\mathrm{i} \neq 1 ; e_{1}, e_{n}$ when $\mathrm{i}=1 ; \mathrm{m}-1$ edges joining the vertex $v_{i}$ in $C_{n}$ to the vertices $w_{i 1}, w_{i 2}, \ldots \ldots, w_{i(j-i)}, w_{i(j+1)}, \ldots \ldots . ., w_{i m} \quad$ in $H_{i} \quad$ viz., $h_{i 1}, h_{i 2}, \ldots \ldots \ldots . ., h_{i(j-1)}, h_{i(j+1)}, \ldots \ldots . . ., h_{i m}$; the edge joining the vertex $v_{i}$ in $C_{n}$ to the vertex $u_{i}$ in $H_{i}$ viz., $h_{i}$; the edge joining the vertex $u_{i}$ in $H_{i}$ to the vertex $w_{i j}$ in $H_{i}$ viz., $l_{i j}$.

Then $\operatorname{adj}(\mathrm{e})=$ number of edges adjacent to e

$$
=2+(\mathrm{m}-1)+1+1=\mathrm{m}+3 .
$$

Theorem 3.2: The edge domination number of

$$
G=C_{n} \odot K_{1, m} \text { is } \mathrm{n} \text {. }
$$

Proof: Consider the graph $G=C_{n} \odot K_{1, m}$ with vertex set V and edge set $\mathrm{E}$, when

$V=\left\{v_{i}, u_{i}, w_{i j}: i=1,2, \ldots \ldots, n ; j=1,2, \ldots \ldots, m\right\}$,

$E=\left\{e_{i}, h_{i}, h_{i j}, l_{i j}: i=1,2, \ldots \ldots, n ; j=1,2, \ldots \ldots, m\right\}$.

Let $D=\left\{h_{1}, h_{2}, \ldots \ldots \ldots, h_{n}\right\}$ where $h_{i}$ is the edge joining the vertex $v_{i}$ in $C_{n}$ to the vertex $u_{i}$ in $H_{i}$. Now edge $h_{i}$ is adjacent to $\mathrm{m}$ edges joining the vertex $u_{i}$ in $H_{i}$ to the vertices $w_{i 1}, w_{i 2}, \ldots \ldots \ldots . . ., w_{i m}$ in $H_{i}$ viz., $l_{i 1}, l_{i 2}, \ldots \ldots \ldots . . ., l_{i m}$; two edges in $C_{n}$ viz., $e_{i-1}, e_{i}$ when $\mathrm{i} \neq 1 ; e_{1}, e_{n}$ when $\mathrm{i}=1$; m edges joining the vertex $v_{i}$ in $C_{n}$ to the vertices $w_{i 1}, w_{i 2}, \ldots \ldots \ldots \ldots, w_{i m}$ in $H_{i}$ viz., $h_{i 1}, h_{i 2}, \ldots \ldots \ldots \ldots, h_{i m}$. That is the edge $h_{i}$ dominates all the edges in $H_{i}$ and two edges in $C_{n}$.

Since this is true for all $\mathrm{i}=1,2, \ldots, \mathrm{n}$, it follows that $\mathrm{D}$ dominates all the edges of G.Thus D becomes an EDS of G. This set is also minimal because, if we delete any edge say $h_{i}$ from $\mathrm{D}$, then the edges in $H_{i}$ are not dominated by any edge in $\mathrm{D}-\left\{h_{i}\right\}$.

We could easily see that any other choice of selection of edges in $C_{n} \odot K_{1, m}$ less than $\mathrm{n}$ will not be an EDS. Hence the edge domination number of $C_{n} \odot K_{1, m}$ is $\mathrm{n}$.

\section{ILLUSTRATIONS MINIMAL EDGE DOMINATING SET}

\section{Theorem 3.2}

The edges with blue colour in Fig. are the edges of minimal edge dominating set.

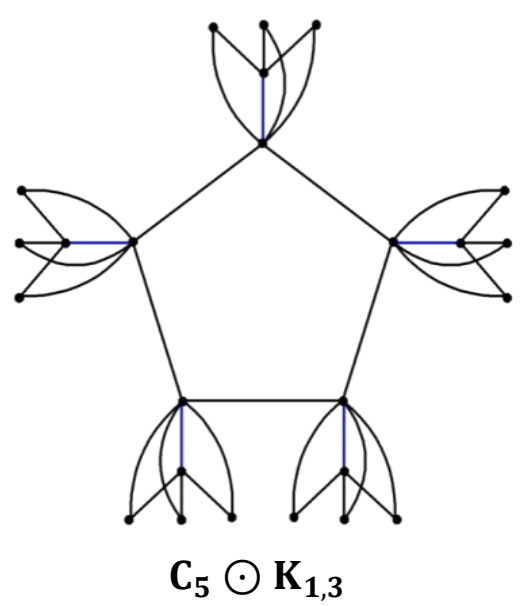

\section{CONCLUSION}

It is interesting to study the edge dominating sets and edge domination number of corona product graph of a cycle with a star. This work gives the scope for an extensive study of other edge dominating sets of this graph.

\section{REFERENCES}

[1].Allan, R.B. and Laskar, R.C. - On domination, independent domination numbers of a graph, Discrete Math., 23, (1978), pp.73- 76.

[2]. Arumugam S., Sithara Jerry - Fractional edge domination in graphs, Appl. Anal. Discrete math.3 (2009), pp.359370 .

[3]. Arumugam S., Velammal S - Edge domination in graphs, Taiwanese Journal of Mathematics, 2 (2) (1998), pp.173179.

[4]. Cockayne, E.J. and Hedetniemi, S.T. - Towards a theory of domination in graphs, Networks, 7, (1977), pp.247 261.

[5]. R. Dutton and W. F. Klostermeyer - Edge dominating sets and vertex covers, Discussions Mathematicae, vol. 33, no.2, (2013), pp.437-456.

[6]. Frucht, R. and Harary, F. - On the corona of Two Graphs, Aequationes Mathematicae, Volume 4, Issue 3, (1970), pp.322 - 325.

[7].Haynes, T.W., Hedetniemi, S.T. and Slater, P.J. Domination in Graphs: Advanced Topics, Marcel Dekker, Inc., New York, (1998).

[8]. Haynes, T.W., Hedetniemi, S.T. and Slater, P.J. Fundamentals of domination in graphs, Marcel Dekker, Inc., New York , (1998).

[9]. Jayaram, S. R - Line domination in graphs, Graphs and Combinatorics, vol. 3, no. 4, (1987), pp. 357-363.

[10]. Kulli, R., Soner, N. D. - Complementary edge domination in graphs, Indian Journal of Pure and Applied Mathematics, vol. 28, no.7, ( 1997 ), pp. 917-920.

[11]. Mitchell S, Hedetniemi, S.T. - Edge domination in trees. Congr. Numer., 19 (1977), pp.489-509.

[12].Yannakakis, M., Gavril, F. - Edge dominating sets in graphs, SIAM Journal on Applied Mathematics, vol. 38, no. 3, (1980), pp. 364-372.

[13]. Zelinka, B. - Edge domination in graphs of cubes, Czechoslovak Mathematical Journal, vol. 52, no. 4, (2002), pp. 875-879. 